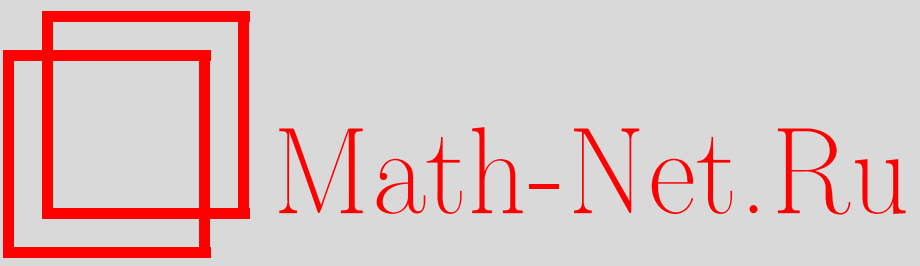

С. Н. Волков, Оптимальное инвестирование с учетом операционных издержек, пропорциональных капиталу портфеля, УМН, 1996, том 51, выпуск 6, 193-194

DOI: https://doi.org/10.4213/rm1023

Использование Общероссийского математического портала Math-Net.Ru подразумевает, что вы прочитали и согласны с пользовательским соглашением

http://www.mathnet.ru/rus/agreement

Параметры загрузки:

IP : 3.81 .55 .215

26 апреля 2023 г., 16:01:19 


\title{
ОПТИМАЛЬНОЕ ИНВЕСТИРОВАНИЕ С УЧЕТОМ ОПЕРАЦИОННЫХ ИЗДЕРЖКЕК, ПРОПОРЦИОНАЛЬНЫХ КАПИТАЛУ ПОРТФЕЛЯ
}

\author{
С.Н. Волков
}

Мы рассматриваем задачу построения оптимальной инвестиционной стратегии при наличии операционных издержек в следующей модели. Пусть финансовый рынок, состоит из банковского счета (облигации):

$$
B_{t}=1
$$

и $d$ рискованных активов (акций), описываемых следующими стохастическими дифференциальными уравнениями:

$$
d S_{t}^{i}=S_{t}^{i}\left(\mu^{i} d t+\sum_{j=1}^{d} \sigma_{i j} d W_{t}^{j}\right)
$$

Здесь $\vec{W}=\left(W^{i}\right)_{1 \leqslant i \leqslant d}-d$-мерный винеровский процесс, $\Sigma=\left(\sigma_{i j}\right)_{1 \leqslant i, j \leqslant d}$ - матрица взаимных ковариаций компонент винеровского процесса.

С математической точки зрения стратегия инвестора на данном рынке определяется как предсказуемый процесс $\Pi=\left(\beta_{t}, \vec{\gamma}_{t}\right)_{t \geqslant 0}$, где $\gamma_{t}^{i}$ (соответственно, $\beta_{t}$ ) есть количество $i$-го рискового (соответственно, безрискового) актива в портфеле в момент времени $t$. Капитал стратегии в момент $t$ равен

$$
V_{t}=\beta_{t}+\langle\vec{\gamma}, \vec{S}\rangle_{t}
$$

(здесь как обычно $\langle$,$\rangle есть скалярное произведение).$

Эквивалентным образом стратегию можно описьвать заданием ее капитала $V$ и вектором пропорций $\left(\alpha_{i}\right)_{1 \leqslant i \leqslant d}$, где

$$
\alpha_{i}=\frac{\gamma^{i} S^{i}}{V}
$$

Мы предполагаем наличие на рынке операционных издержек, пропорцианальных капиталу портфеля, т.е. за покупку или продажу акций взимается плата в размере $\eta V$. В этом случае условие самофинансируемости принимает следующий вид:

$$
d V_{t}=V_{t}\left[\langle\vec{\alpha}, \vec{\mu}\rangle d t+\left\langle\vec{\alpha}, \Sigma d \vec{W}_{t}\right\rangle-\eta d N_{t}\right],
$$

где $\left(N_{t}\right)_{t \geqslant 0}=\sum_{s \leqslant t} I\left(\left|\Delta \vec{\gamma}_{s}\right|>0\right)$ - считающий процесс “воздействий” инвестора.

В работе А. Мортона и С. Плиски [1] рассматривалась задача максимизации асимптотической ставки роста инвестиционного портфеля:

$$
\lim _{T \rightarrow \infty} \frac{E\left[\ln \left(V_{T} / V_{0}\right)\right]}{T} \rightarrow \max \stackrel{\text { def }}{=} \Phi_{\infty}(\eta)=\Phi_{\infty}(\eta, 0) .
$$

Было показано, что (1.3) сводится к решению задачи Стефана с подвижной границей и в случае $d=1$ было дано явное решение.

В случае $d>1 \mathrm{~K}$. Аткинсон и П. Уилмотт [2] с помощью "метода возмущений" нашли решение задачи Стефана в первом приближении по $\eta$.

В настоящей работе рассматривается следующая задача максимизации средней доходности инвестиционного портфеля на конечном временном интервале $[0, T]$ в случае $\eta \approx 0$ :

$$
E R(\beta)=\frac{1}{\beta T}\left(E\left[V_{T} / V_{0}\right]\right)^{\beta}-1 \rightarrow \max \stackrel{\text { def }}{=} \Phi_{T}(\eta, \beta), \quad \beta \in(-\infty, 1) .
$$

В пределе $\beta \rightarrow 0$ получаем аналог задачи (1.3) на конечном временном интервале. 
Пусть $R^{*}(\beta)=R^{*}(\eta, \beta)$ есть максимальная средняя доходность самофинансируемых портфелей определенная в (1.4). Случай $\eta=0$ отсутствия операционных издержек был изучен Р. Мертоном [3]. Его резултат можно переформулировать с учетом введенных обозначений следующим образом:

где вектор

$$
R^{*}(0, \beta)= \begin{cases}\frac{1}{\beta T}\left(\exp \frac{\beta\left\langle\vec{\alpha}^{*}, \vec{\mu}\right\rangle}{2}-1\right), & \beta \in(-\infty, 1), \beta \neq 0, \\ \frac{\left\langle\vec{\alpha}^{*}, \vec{\mu}\right\rangle}{2 T}, & \beta=0,\end{cases}
$$

$$
\vec{\alpha}^{*}=\vec{\alpha}^{*}(\beta, 0)=\frac{1}{1-\beta}\left(\Sigma \Sigma^{T}\right)^{-1} \vec{\mu}
$$

определяет пропорцию рискованной части капитала в оптимальном инвестиционном портфеле (так называемая "точка Мертона").

Введем следующие обозначения: $E$ - единичная матрица, $I$ - матрища размера $d \times d$, все элементы которой равны $1, \operatorname{Diag}\left(\vec{\alpha}^{*}\right)$ - диагональная матрица, элементы которой равны соотвествующим элементам вектора $\alpha^{*}$.

ПРЕДЛОЖЕнИЕ 1. 1) Имеет место асимптотическое представление:

$$
R^{*}(\eta, \beta)=R^{*}(0, \beta)-\eta^{1 / 2} v^{*}\left(1+R^{*}(0, \beta) \beta T\right)+o\left(\eta^{1 / 2}\right),
$$

əде

$$
v^{*}=2 \operatorname{tr}(B M), \quad B=\left\langle\Sigma \operatorname{Diag}\left(\vec{\alpha}^{*}\right)\left(E-I \operatorname{Diag}\left(\vec{\alpha}^{*}\right)\right), \Sigma \operatorname{Diag}\left(\vec{\alpha}^{*}\right)\left(E-I \operatorname{Diag}\left(\vec{\alpha}^{*}\right)\right)\right\rangle
$$

а матрица $M$ является решением следующего уравнения

$$
8 M B M+4 \operatorname{tr}(B M) M=(1-\beta) \Sigma^{T} \Sigma .
$$

2) Стратегия П у которой изменение структуры портфеля осуществляется только в те моменты времени, когда отклонение пропорции рискованной части капитала от вектора (1.5) $\Delta \vec{\alpha}=\vec{\alpha}-\vec{\alpha}^{*}$ удовлетворяет соотношению

$$
\langle\Delta \vec{\alpha}, M \Delta \vec{\alpha}\rangle \geqslant \eta^{1 / 4}
$$

причем в количестве необходимом для восстановления $\vec{\alpha}$ в точку $\vec{\alpha}^{*}$, является асимптотически оптимальной:

$$
R^{\widetilde{\Pi}}(\eta, \beta)=R^{*}(0, \beta)-\eta^{1 / 2} v^{*}\left(1+R^{*}(0, \beta) \beta T\right)+o\left(\eta^{1 / 2}\right)
$$

ЗАмЕЧАНИЕ. Отметим что указанная оптимальная стратегия совпадает с асимптотически оптимальной стратегией Аткинсона-Уилмотта [2].

Основная идея в доказательстве предложения 1 состоит в использовании свойства автомодельности оптимизационной задачи, а также эргодической теоремы для управляемых случайных процессов, позволяющей перейти от задачи на конечном временном интервале к задаче на бесконечном временном интервале.

Автор выражает благодарность Д. О. Крамкову за полезные обсуждения и замечания.

\section{СПИСОК ЛИТЕРАТУРЫ}

[1] Morton A. J., Pliska S.R. Optimal Portfolio Management with Fixed Transaction Costs // Math. Finance. 1995. V. 5. № 4. P. 337-356. [2] Atkinson C., Wilmott P. Portfolio Management with Transaction Costs: An Asymptotic Analysis of the Morton and Pliska Model // Math. Finance. 1995. V. 5. № 4. P. 357-367. [3] Merton R. C. Lifetime Portfolio Selection under Uncertainty. The Continuous Time Case // The Review of Economics and Statistics. 1989. V. 51. № 3. P. 247-257. 